\title{
Halo Studies for the ESS and Linac4 Front-Ends
}

\author{
Frank Gerigk \\ ISIS Accelerator Theory \& Future Projects Group \\ CCLRC Rutherford Appleton Laboratory, Chilton, Didcot, Oxon, U.K.
}

\begin{abstract}
In high intensity proton linacs, beam halo can be observed as early as the very first stages of acceleration. The inital population of halo particles found in the output distribution of the RFQ, increases in chopper lines, which interrupt the regular focusing pattern of RFQ and DTL. The resulting emittance growth and halo development contributes to beam loss in the low-energy accelerator sections and partly defines the beam quality in the high-energy sections. U sing the examples of the ESS and Linac4 (CERN) front ends (RFQ to $20 \mathrm{M} \mathrm{eV}$ ) we investigate emittance grow th and beam hal o due to statistically distributed field and gradient errors.
\end{abstract}

\section{INTRODUCTION}

The analysis of statistical errors represents the fi nal stage in every linac design. Emittance growth, phase $\&$ energy jitter and beam loss due to these errors defi ne the tolerances in the machine and thus have a direct impact on the project costs.

This paper reports on the progress in simulating statistical errors in the ESS [1] and Linac4 [2] front-ends, both high-intensity $\mathrm{H}^{-}$linacs with a beam chopper in their $M$ edium E nergy B eam Transport (M EBT) line. The simulation approach using IM PACT [3] is outlined, and results from the simulation of quadrupole gradient errors as well as RF phase and gradient errors are reported. $\mathrm{Fi}$ nally the resul ts are interpreted with respect to the current understanding of halo development.

\section{SIMULATION}

Lattices. B oth front-ends start with a simulated RFQ output distribution [4] followed by a M EBT containing a beam chopper with several focusing elements. Two Alvarez Drift Tube Linac (DTL) tanks then raise the energy from 2.5 to $20 \mathrm{M} \mathrm{eV}$ in the ESS case and from 3.0 to $25 \mathrm{MeV}$ for Linac4. Long term effects will be visualized using the full Linac4 lattice up to an energy of $120 \mathrm{M} \mathrm{eV}$. The lattice parameters are summarized in Table 1.

Conventions. In order to quantify the amount of halo in a distribution snapshot, the fraction of particles outside $n$ times its r.m.s. emittance (in each plane) is plotted. Thus the development of beam halo can be clearly separated from $\varepsilon_{r . m . s .}$ growth and/or density oscillations in
TABLE 1. Lattice parameters

\begin{tabular}{l|cc}
\hline & ESS & Linac4 \\
\hline RFQ output & $2.5 \mathrm{M} \mathrm{eV}$ & $3.0 \mathrm{M} \mathrm{eV}$ \\
peak current & $57 \mathrm{~mA}$ & $30 \mathrm{~mA}$ \\
RF frequency & $280 \mathrm{M} \mathrm{Hz}$ & $352 \mathrm{M} \mathrm{Hz}$ \\
\hline MEBT & $3.11 \mathrm{~m}$ & $3.73 \mathrm{~m}$ \\
no. of chopper plates & 4 & 2 \\
no. of buncher cav. & 6 & 3 \\
no. of quadrupoles & 13 & 11 \\
\hline DTL & $11 \mathrm{~m}$ & $9 \mathrm{~m}+6.1 \mathrm{~m}^{*}$ \\
no. of RF sources & 2 & $2+1$ \\
no. of gaps & 77 & $82+29$ \\
no. of quadrupoles & 78 & $83+29$ \\
\hline output energy & $20.3 \mathrm{M} \mathrm{eV}$ & $24.9 \mathrm{M} \mathrm{eV}$ \\
\hline CCDTL & & $47.6 \mathrm{~m}$ \\
no. of R F sources & & 10 \\
no. of 3/4-gap tanks & & 37 \\
no. of quadrupoles & & 37 \\
\hline output energy & & $120 \mathrm{M} \mathrm{eV}$ \\
\hline * + refers to the full Linac4 & & \\
\hline & &
\end{tabular}

real space. A n example is shown in Fig. 1 where the two input distributions are plotted. Both distributions carry particles with core radii of up to 5 times the r.m.s. value. A uniform error distribution is used for all simulations, meaning that the r.m.s. errors can be obtained by multiplying the quoted max. error amplitudes by $\approx 0.58$.

Simulation details. A preprocessor creates $m$ random error sets, applies them to the original lattice and stores the new input fi les in scratch directories. With a simple shell script the jobs are then submitted to a L inux 


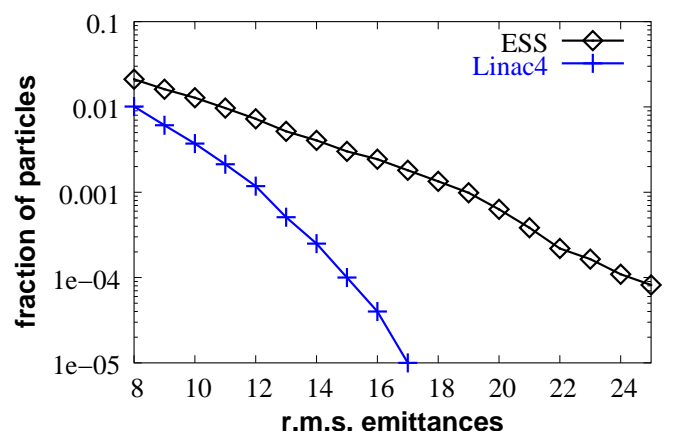

FIGURE 1. Transverse halo: particles outside $n \times \varepsilon_{\text {r.m.s. }}$ in the input distributions for the ESS and Linac4 front-ends.

cluster. For the full Linac4, 500 error sets with 50000 particles are simulated for each error amplitude, using $\approx$ 14 hours of CPU time on 30 processors. For the shorter front-end simulations 300 error sets are created. The process is then repeated for each error amplitude and evaluated by a post-processor module.

\section{STATIC \& DYNAMIC ERRORS}

In the following we use the classic distinction between 'dynamic' errors that change from pulse to pulse or within single RF pulses and 'static' errors that change very slowly (seasonal changes) or remain completely unchanged during operation [5], [6]. Static errors are generally a residue of the initial adjustment of the lattice elements, e.g. random (gap to gap) RF amplitude errors from the fi eld adjustment with bead pull $(\approx 1 \%)$, or random quadrupole gradient errors $(\approx 1 \%)$. The same category applies for mismatch and alignment errors which are not covered in this paper. While the static errors usually change from element to element the dynamic errors tend to be grouped, originating from pulse to pulse variations of $\operatorname{RF}\left(<1 \%, 1^{\circ}\right)$ or quadrupole $(<0.5 \%)$ power supplies.

Figure 2 shows the average $\varepsilon_{\text {r.m.s. }}$ growth rates with respect to maximum transverse and longitudinal error amplitudes for grouped or completely random errors. In the transverse plane the grouped and ungrouped errors result in the same average $\varepsilon_{r . m . s .}$ growth rates, cutting in half the number of simulations needed to evaluate both error sources. In the longitudinal plane, however, the grouped errors yield larger $\varepsilon_{\text {r.m.s. }}$ growth (and larger phase $\&$ energy jitter) than the ungrouped errors. Although modern RF power sources with temperature controlled cables provide a margin of $0.5 \%$ fi eld error and $0.5^{\circ}$ phase error during the pulse, the error margins for the fi rst bunches of a pulse may be considerably higher [5] and thus become a major concern.
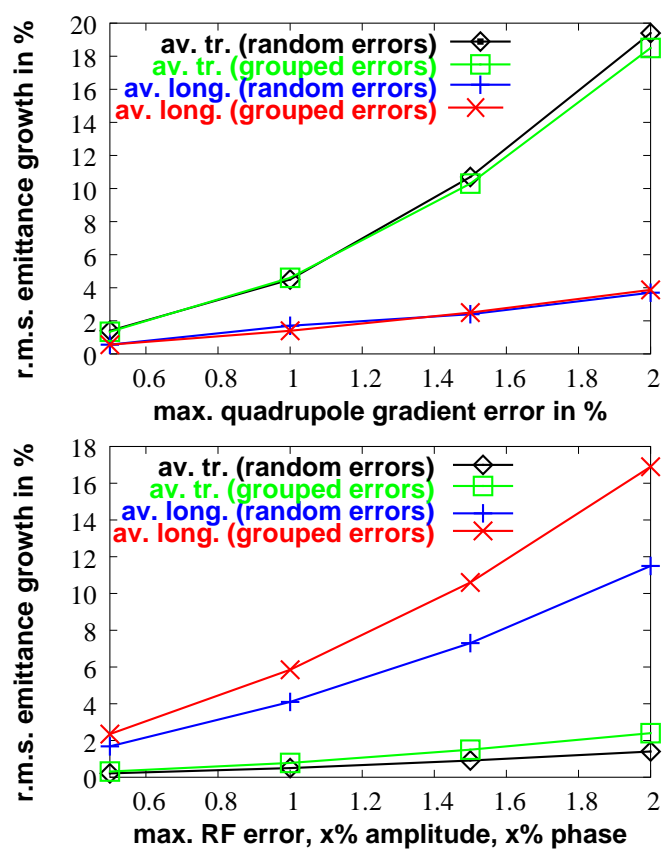

FIGURE 2. Upper graph: average $\varepsilon_{\text {r.m.s. }}$ increase for dynamic (grouped) and static (random) quadrupole gradient errors in the full Linac4; lower graph: dynamic and static RF field and phase errors

The average $\varepsilon_{r . m . s .}$ growth for realistic dynamic and static error margins seems to be very small and therefore of no concern for low-loss machine operation. N evertheless, a combination of all static and dynamic errors including alignment errors and mismatch may easily yield unacceptable losses and has to be evaluated for each particular machine.

In both planes, the maximum $\varepsilon_{\text {r.m.s. }}$ growth rates for the worst cases are $\approx 3$ to 4 times higher than for the plotted average cases. For dynamic errors the worst cases are of little importance since normal machine operation will automatically yield the average. The same reasoning applies for certain static errors (e.g. quadrupole gradient errors) which can be reduced with automated tuning systems. Some static errors, however, cannot be compensated (e.g. residual fi eld adjustment errors in multi-gap RF tanks) and for those the worst case results should be taken into account.

\section{PHASE \& ENERGY JITTER}

The dynamic errors in the longitudinal plane not only result in $\varepsilon_{\text {r.m.s. }}$ growth but also in phase and energy deviation (jitter) from the nominal trajectory. If the linac beam is injected into a subsequent accelerator or a transport line with RF cavities, the phase and energy jitter has 
to be limited to fi t into the RF bucket of the following system.

We fi nd that the largest phase $\&$ energy deviations usually originate from transitions between sections that are powered by different amplifi ers and/or have different focusing structures. Opposite error amplitudes at these transitions can provide large kicks which are then amplifi ed in subsequent drifts. A $n$ example is shown in Fig. 3, where the four worst case energy deviations are plotted for the two front-ends.
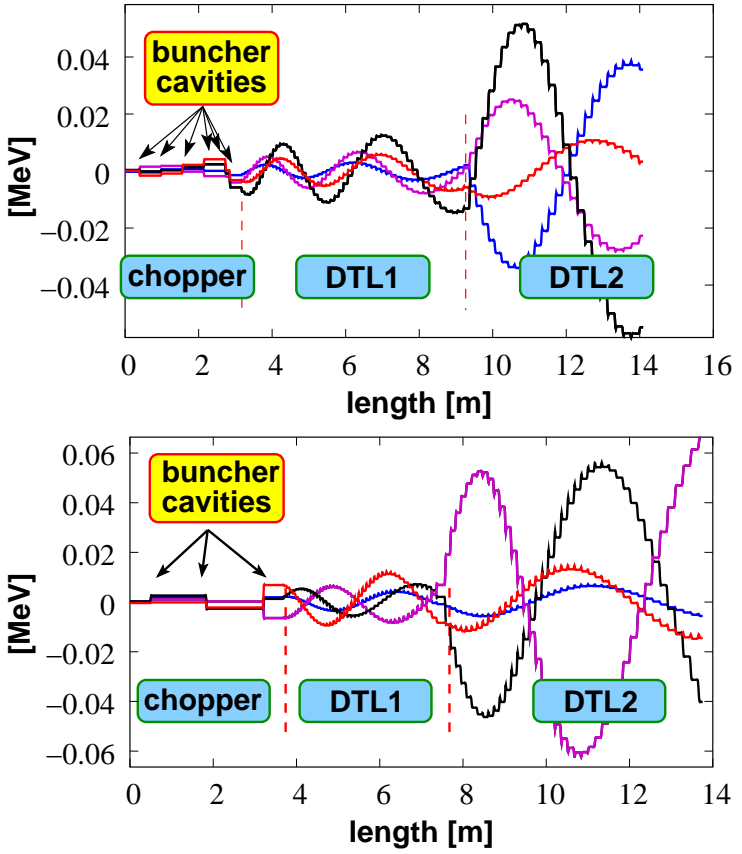

FIGURE 3. The four worst case energy deviations for a maximum of $1 \%$ field error and $1^{\circ}$ phase error in the ESS (upper graph) and Linac4 (lower graph) front-end.

The average and maximum phase and energy jitter at the end of Linac4 are between 15 and 30\% higher than for the ESS. This can probably be explained by the more compact design of the ESS MEBT (see Table 1) and the 'missing gap' at the transition of DTL1 and DTL 2 in L inac4. In the ESS front-end, the two DTL tanks have a 'seamless' transition.

Considering Fig. 2 and 3 it is obvious that the partitioning of RF power supplies and cavities plays a crucial role in the development of phase $\&$ energy jitter. In the following example the jitter at the end of Linac4 is plotted for its original partitioning of power supplies and RF cavities, and for a partitioning with a reduced number of amplifi ers (5 instead of 16, Fig. 4).

Contrary to the results with grouped and ungrouped RF errors in Fig. 2, we fi nd that the lower number of power supplies results in a much lower phase $\&$ energy jitter and a much reduced average longitudinal $\varepsilon_{\text {r.m.s. }}$. growth $(2.6 \%$ instead of $5.8 \%)$. This suggests that the power
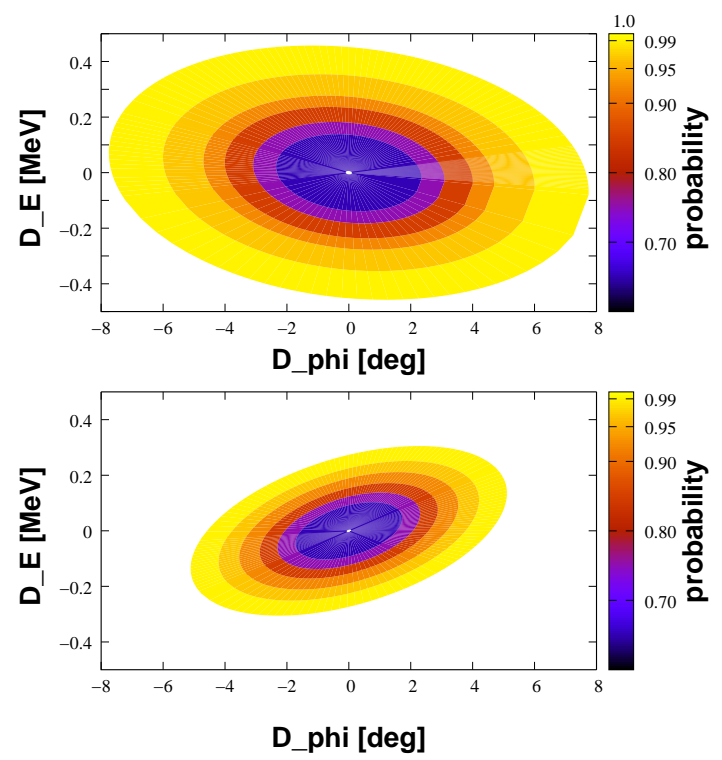

FIGURE 4. Phase $\&$ energy jitter at the Linac4 output for a maximum of $1 \%$ field error and $1^{\circ}$ phase error using 16 (upper graph) or $\mathbf{5}$ (low er graph) RF amplifiers.

splitting should be carefully optimized for each machine and that the optimum number of RF gaps per power source may be different for different lattice types and different energies.

\section{HALO FROM STATISTICAL ERRORS?}

Parametric beam halo devel ops for integer ratios (usually 2:1) between the oscillations of a mismatched beam core and the oscillations of single particles. This type of coherent core oscillation can be excited by initial mismatch and can remain remarkably stable throughout an entire linac. Figure 5 shows one such example for a fast-mode

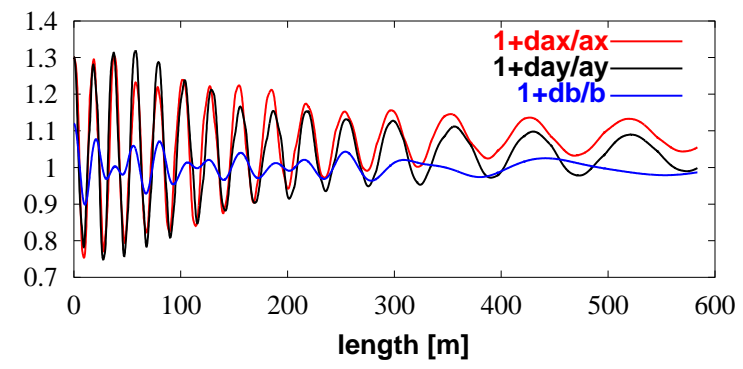

FIGURE 5. 30\% fast mode excitation in the superconducting section of the SPL.

excitation in the $600 \mathrm{~m}$ long superconducting section of the SPL [7]. Energy transfer from the core oscillations to the single particle orbits eventually damps the oscillations of the core and thus creates halo. 
Similar oscillations, though much more irregular, can be observed in simulations with statistical errors, e.g. in Fig. 6 where the radial deviations for an average case of quadrupole errors ( $\max . \pm 1 \%$ ) reach amplitudes between 5 and $10 \%$. For the worst case they increase to $40 \%$, reaching similar levels to those generated by strong initial mismatch. Figure 6 also shows that the worst ra-
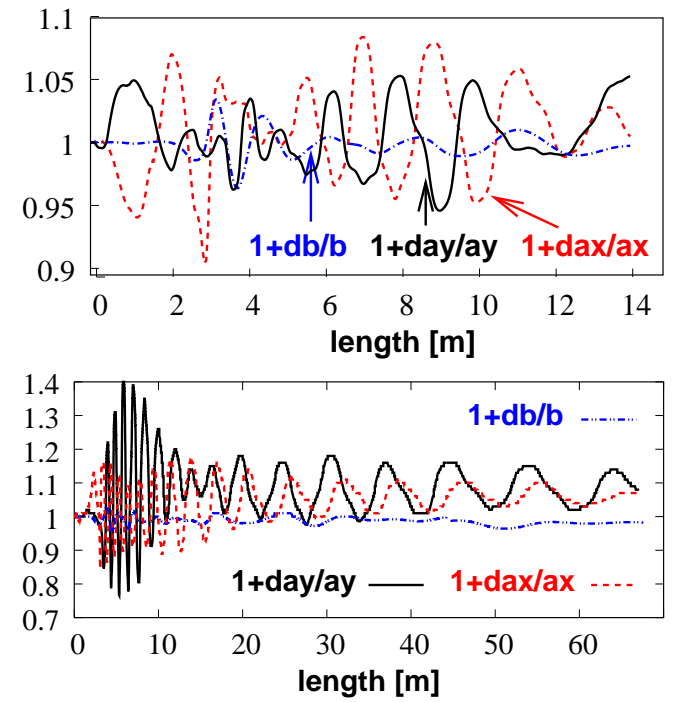

FIGURE 6. Upper graph: average case of radial deviations for $\pm 1 \%$ quadrupole gradient errors in the ESS front-end $(\approx$ $5 \% \varepsilon_{r . m . s .}$ growth), lower graph: worst case for Linac4 $(\approx$ $100 \% \varepsilon_{\text {r.m.s. }}$ growth).

dial deviations occur after the MEBT, in the fi rst two DTL tanks. Unless these tanks are designed with large bore radii they are likely to suffer from high beam loss. The corresponding beam halo for the ESS front-end (Fig. 7) shows an almost invisible increase for the average case and roughly a doubling of large amplitude particles for the worst case. For the full L inac4, Fig. 8 shows a more visible effect for the average error case.

Despite the diffi cult interpretation of these curves (beam halo al ready for the matched case, particle loss, low number of particles $\leq 50000$, etc.) it seems as if statistical errors might have the potential to create beam halo. Fig-

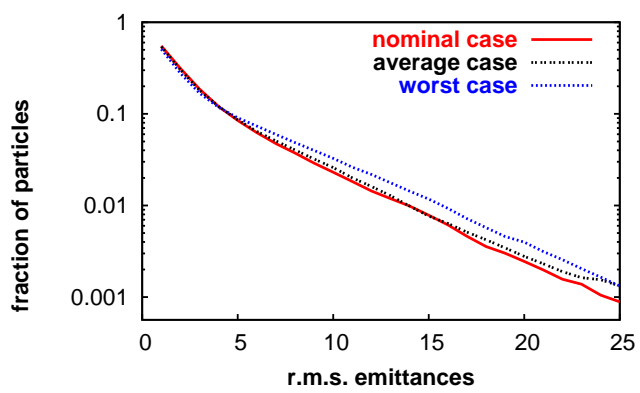

FIGURE 7. Transverse halo due to ungrouped $1 \%$ (max.) quadrupole gradient errors in the ESS front-end. ures 7 and 8 both show an increased number of particles beyond the core emittance $\left(>5 \cdot \varepsilon_{r . m . s .}\right)$ even though the amount of the observed halo is less dramatic than for initial mismatch.

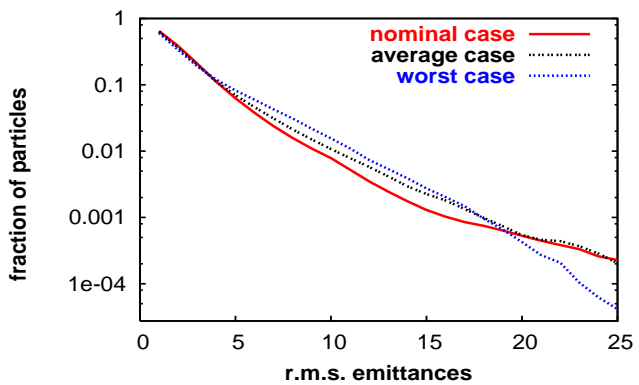

FIGURE 8. Transverse halo due to ungrouped 1\% (max.) quadrupole gradient errors in Linac4.

\section{SUMMARY AND OUTLOOK}

First results of IMPACT simulations of dynamic and static gradient \& phase errors were presented for L inac4 and the ESS front-end. Quadrupole gradient variations for grouped and ungrouped errors resulted in equal $\varepsilon_{r . m . s}$. growth rates and seem to be of little concern. Longitudinally the partitioning of power sources and RF gaps had a signifi cant influence on the resulting $\varepsilon_{\text {.m.s. }}$. growth and energy $\&$ phase jitter. A dditional concerns are the possibility of an unfortunate combination of residual fi eld adjustment errors, and the enhancment of RF error bars for the fi rst bunches of an RF pulse. The simulations indicate the possibility of halo development due to statistical errors, although more systematic work is needed to understand the process fully. A combination of all error sources and an evaluation of alignment errors has yet to be done and might add signifi cance to the effects of single error sources.

\section{REFERENCES}

1. Gerigk, F., Revised ESS Front-End (2.5 - $20 \mathrm{MeV})$, Tech.Rep. (2003).

2. Gerigk, F., and V retenar, M., "Design of a $120 \mathrm{M} \mathrm{eV}$ $\mathrm{H}^{-}$Linac for CERN High-Intensity Applications," in Proceedings LINACO2, K yongiu, Korea, 2002.

3. Qiang, J., Ryne, R. D., H abib, S., and Decyk, V., Journal of Computational Physics, 163, 1-18 (2000).

4. The ESS Project Volume III, Technical Report, ISBN 3-89336-303-3, 2002.

5. V retenar, M., private communications (2003).

6. Findlay, D., private communications (2003).

7. V retenar, M., editor, Conceptual Design of the SPL, a High-Power Superconducting $\mathrm{H}^{-}$Linac at CERN, CER N 2000-012, 2000. 